\title{
Yaël Dagan, La NRF entre guerre et paix. 1914-1925
}

\section{Gian Luigi Di Bernardini}

\section{(2) OpenEdition}

\section{Journals}

\section{Edizione digitale}

URL: http://journals.openedition.org/studifrancesi/7691

DOI: 10.4000/studifrancesi.7691

ISSN: 2427-5856

\section{Editore}

Rosenberg \& Sellier

\section{Edizione cartacea}

Data di pubblicazione: 1 décembre 2009

Paginazione: 662

ISSN: 0039-2944

\section{Notizia bibliografica digitale}

Gian Luigi Di Bernardini, «Yaël Dagan, La NRF entre guerre et paix. 1914-1925», Studi Francesi [Online], 159 (LIII | III) | 2009, online dal 30 novembre 2015, consultato il 08 janvier 2021. URL: http:// journals.openedition.org/studifrancesi/7691 ; DOI: https://doi.org/10.4000/studifrancesi.7691

Questo documento è stato generato automaticamente il 8 janvier 2021.

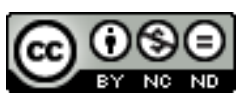

Studi Francesi è distribuita con Licenza Creative Commons Attribuzione - Non commerciale - Non opere derivate 4.0 Internazionale. 


\title{
Yaël Dagan, La NRF entre guerre et paix. 1914-1925
}

\author{
Gian Luigi Di Bernardini
}

\section{NOTIZIA}

YAËL DAGAN, La NRF entre guerre et paix. 1914-1925, Paris, Tallandier, 2008, pp. 427.

1 Le volume de Yaël Dagan revient sur une phase-clé de l'histoire de «La Nouvelle Revue Française», la période 1914-1925, pendant laquelle ses fondateurs doivent d'abord se confronter à l'éclatement de la Grande Guerre, ensuite à la difficile paix qui suit.

Dagan dresse, dans Mobilisation et sacrifices (pp. 39-88), un panorama des différentes attitudes de -Gide, Schlumberger, Copeau, etc., à travers la lecture de leurs journaux intimes, de leurs correspondances, mais aussi des articles publiés dans la revue. On lit aisément la fascination de ce groupe (bien qu'il ne forme pas un bloc unitaire) pour la mobilisation et la rhétorique patriotique; cet attrait est reflété par un lexique relevant de la sphère mystique et religieuse, issu d'une réflexion sur l'éthique du devoir et du sacrifice diffusée pendant la décennie précédente et qui est également à la base de l'esthétique classiciste de la revue.

3 Le groupe de la «NRF», comme d'ailleurs toute la France, est investi par la crise de démobilisation intellectuelle de 1917, que Dagan analyse dans Crises (pp. 89-140): crises publiques (on s'interroge sur l'orientation à donner à la «NRF» à sa reparution) aussi bien que personnelles (la crise religieuse qui pousse un moment -Gide vers la conversion en 1916).

Remobilisation et victoire (pp. 141-181) concerne la difficulté de sortir de l'atmosphère envenimée de la guerre, surtout au niveau idéologique. L'Allemand de Jacques Rivière, par exemple, insiste sur une peinture stéréotypée du peuple allemand, décrit comme barbare, non cultivé et, donc, comme le mal absolu du continent européen.

Dans Nouveau départ (pp. 183-225), on explore les orientations assumées par la «NRF» lors du lancement de sa nouvelle série, synthétisées dans un article-manifeste de 
Jacques Rivière qui provoque de nombreuses réactions parmi les collaborateurs de la revue.

6 La Revue de Rivière (pp. 227-269) décrit les structures et les contenus de la nouvelle série de la «NRF», ainsi que le style de la direction de Jacques Rivière jusqu'à 1925, l'année de sa mort. Il s'agit d'une période féconde pour la revue qui double (et même triple) ses abonnements, surtout à l'occasion de la publication de certains textes importants (Proust, par exemple, ensuite Benda, etc.); en même temps, la maison d'édition Gallimard s'institutionnalise et l'équipe des collaborateurs se renouvelle presque complètement. La «NRF» assume rapidement une importance nationale (et même internationale) qui lui vaut un prestige grandissant.

7 Le dernier chapitre, De la guerre à la paix (pp. 271-330), s'occupe surtout de la période après 1923. La revue prône l'idée d'une unité européenne qui contraste les nationalismes aussi bien que les internationalismes.

8 Dans l'Épilogue (pp. 331-340), Dagan donne des aperçus sur l'âge d'or de la «NRF», sans cacher par exemple une certaine difficulté à s'adapter (idéologiquement) à la nouvelle montée de la tension avec l'Allemagne. 